\title{
sciendo
}

\section{Turing Patterns and Biological Explanation}

\author{
Maria Serban \\ University of Copenhagen \\ BIBLID [0873-626X (2017) 47; pp. 529-552] \\ DOI: $10.1515 /$ disp-2017-0017
}

\begin{abstract}
Turing patterns are a class of minimal mathematical models that have been used to discover and conceptualize certain abstract features of early biological development. This paper examines a range of these minimal models in order to articulate and elaborate a philosophical analysis of their epistemic uses. It is argued that minimal mathematical models aid in structuring the epistemic practices of biology by providing precise descriptions of the quantitative relations between various features of the complex systems, generating novel predictions that can be compared with experimental data, promoting theory exploration, and acting as constitutive parts of empirically adequate explanations of naturally occurring phenomena, such as biological pattern formation. Focusing on the roles that minimal model explanations play in science motivates the adoption of a broader diachronic view of scientific explanation.
\end{abstract}

\section{Keywords}

Mathematical models, development, patterns, biological explanation.

\section{Introduction}

The epistemology of mathematical modeling in the biological sciences generates difficult questions for both practicing scientists and philosophers. Mathematical models are typically assigned one or more of the following functions in biological research: unification, model fitting, mechanism identification, and prediction (Winther 2012, Brigandt 2013). Less agreement exists over whether mathematical models play a leading role in biological explanation and how that role should be understood (Fox Keller 2003). Philosophical analyses themselves diverge as to whether we should understand math- 
ematical models as intermediary stages towards causal explanations or whether we should make room for distinctively mathematical explanations of empirical phenomena (e.g. Baker 2005, 2009; Pincock 2007, 2011, 2012, 2015; Lange 2013). This divide is also reflected in the normative judgments made by scientists working within different methodological traditions (cf. Fox Keller 2003; Amundson 2005).

How we answer the question about the explanatory roles of mathematical models in biological research will vary depending on the class of models chosen and on their intended applications in specific research contexts. I do not aim to offer a blanket thesis about mathematical explanation or model explanation across the life sciences. Instead, I focus on a particular type of minimal mathematical modelling, aiming to identify its distinctive features and its role in a special class of explanations in developmental biology, viz. morphological explanations (Nathan 2012). If this analysis is on the right track, it illustrates the virtues of adopting a diachronic and functionalist perspective on the general problem of model-based explanation.

The example that will help motivate and pin down some of the features of my thesis about the link between mathematical modeling and explanation is Turing's reaction-diffusion model of the chemical basis of morphogenesis (Turing 1952). The central idea of the model is that biological patterning is a self-organizing phenomenon in which the interaction of two stabilizing processes gives rise to instabilities that in turn produce the spatial patterns observed in the course of development. In its simplest form, the model is expressed as a set of coupled reaction-diffusion equations which describe the ways in which cells differentiate in a concentration-dependent manner in response to a chemical pre-pattern.

Turing's work on pattern formation has triggered new research programs in both mathematics and biology. In the mathematical domain, the focus fell on exploring the rich variety of behaviours of the system of nonlinear parabolic equations, while theoretical and experimental work in biology targeted the discovery and detailed analysis of the structure and function of morphogens. The following discussion examines a range of studies where Turing reaction-diffusion (RD) models have played a critical role. The aim is to develop novel philosophical ideas about the epistemology of mathematical modelling which will complement and extend current philosophical analyses 
of biological research practices (e.g. Fox Keller 2003, Bechtel and Richardson 2010).

There are two parts of my use of Turing's pioneering work in mathematical biology (cf. Fox Keller 2003, Murray 2003, Bourgine and Lesne 2006, Maini 2012). For the first part, I emphasize two distinctive epistemological features of Turing's original modeling technique: (1) the target of Turing's mathematical model is described in broadly fictional terms; otherwise put, the features represented in the mathematical model are fictional or imaginary constructions based on a series of simplifying and idealizing assumptions (Levy 2015). (2) Turing's use of the model illustrates clearly the inferential link between the use of rigorous mathematical techniques for stability analysis and the discovery or postulation of the causal processes responsible for observable features or phenomena. Focusing on these features will help make explicit the structure of the biological explanations based on this modelling technique and to discuss their causal status. The second part of my analysis relies on a brief overview of the recent applications of Turing models in different areas of developmental biology in order to show how a diachronic and functionalist perspective can account for the extension and transformation of reaction-diffusion based explanations in developmental biology.

I start with a brief and informal discussion of Turing's mathematical modelling and of the types of questions it opens up for investigation. A selective survey of the current extensions and biological applications of Turing-models will provide a launchpad for a more abstract analysis of the interplay between mathematical modeling, biological theorizing, and experimentation. I argue that in this kind of setup it is possible to identify and articulate the explanatory contributions of this class of mathematical models to understanding the origin, stability and robustness of biological patterns.

\section{Turing patterns}

Turing patterns are spatial or spatio-temporal organizational features observed in the physical and biological world: the shapes of sand dunes, the intricate patterns on a sunflower, the number and distribution of arthropod appendices, animal coat markings, and so on. In the biological domain these patterns or regular shapes are 
typically manifested at the tissue level. Different types of theoretical and experimental approaches have been devised to explain the richness and variety of biological patterns. In particular, developmental and evolutionary biology aim to explain why and how these types of patterns arise.

The chemical basis of morphogenesis published by Alan Turing in 1952 proposed a possible answer to the question of how a tissue can be patterned. Turing conjectured that cells respond to a chemical pre-pattern. He coined the term morphogen for a chemical to which cells would respond by differentiating in a concentration-dependent way. In order to generate spatial patterns in a chemical system, he considered a system of morphogens reacting and diffusing in such a way that, in the absence of diffusion, they produced a spatially uniform steady state which would also be stable. He then proved that by introducing diffusion into the system, one obtains instabilities that in turn lead to heterogeneous patterns of chemical concentrations. The surprising character of the results of Turing's model becomes obvious when one considers the assumptions on which it was built. Turing took the reaction kinetics governing his system to be stabilizing. In addition, diffusion was known to be a stabilizing process in the sense that it homogenises spatial patterns. The unexpected result consisted in proving mathematically that the interaction of two stabilising processes can produce an instability (now known as diffusiondriven instability, cf. Murray 2003, Maini 2012).

\subsection{Elements of a modelling strategy}

It will be helpful to spell out a bit more Turing's modelling strategy and mathematical techniques. The following presentation will remain largely informal, highlighting the main stages or components in the development of this modeling process. We can identify Turing's starting point with the following question: how to account for the transformation of an initially perfectly symmetric embryo (represented in the model as a sphere) into a structured organism? Turing recasts this as the more general problem of the formation of patterns from an initial homogeneous state (Turing 1952). This phenomenon is also known as symmetry breaking. It might seem odd to talk of symmetry breaking in the case of Turing patterns given their spatial peri- 
odicity and organization into stripes and spots that respect the geometry of the (biological) system. However this initial puzzlement can be easily discarded by noting that the symmetry breaking is defined in terms of the perfect or complete symmetry of the system in its homogeneous state (i.e., invariant under all translations, rotation, reflexions, inversions, and extensions). Mathematically, the symmetry breaking is reflected in the fact that the solution of the dynamical equation has less symmetries than the equation describing the original state of the system.

Turing offers the following account of symmetry breaking: random perturbations of the homogeneous, perfectly symmetric state of the system will develop into very different states. Some of them will be cancelled out, while others will be amplified. The intrinsic dynamics of the system will thus select some fluctuations whose characteristics will be reflected in the final solution. Other fluctuations will not have any effect upon the final state of the system. The behaviour of the system could in fact be modelled as other resonance phenomena by the study of a pendulum or of an oscillator. It is also important to point out that Turing recognized that the initial perturbation of the system from its equilibrium state does not determine the characteristic features of the resulting patterns, but only the possibility of their production. The intrinsic instability of the dynamics determines the features of the different types of patterns produced. Thus, according to Turing's model, the formation of patterns of morphogens is spontaneous - it does not require any predetermined pattern nor an external design or blueprint.

The dynamics that Turing modeled mathematically is the result of the interaction between various chemical reactions and the diffusion of reactants, as suggested above. The Turing patterns thus correspond to spatially periodic variations of the concentrations of the different chemical substances reacting in a given situation. In the simplest case one assumes: an auto-activating substance A that also activates the production of another substance B; substance B inhibits the production of $\mathrm{A}$, and $\mathrm{B}$ diffuses more quickly than $\mathrm{A}$. Under these assumptions, a small local fluctuation can induce a small excess of the activator A which in turn accelerates the production of both $\mathrm{A}$ and $\mathrm{B}$. The excess of $\mathrm{B}$, which also diffuses more quickly than $\mathrm{A}$, creates an inhibiting barrier around the initial point, isolating the 
concentration of $\mathrm{A}$ with a region richer in $\mathrm{B}$. In order to arrive at spatial patterns, one needs to consider several iterations of this process. At a certain distance from the first fluctuation, another similar fluctuation can develop, creating another A region, and so on. The dynamically most stable partition of activator, determined by the selective amplification of the many small fluctuations that affect spontaneously the state of the homogeneous mix of the two substances forms a periodic pattern.

Turing talks of stationary waves in order to designate this pattern, but the term might be misleading since it suggests incorrectly that the dynamics of the RD systems is the same as that of the equations describing sound or light propagation. However, Turing is explicit about the fact that he models these molecular mechanisms at the macroscopical scale, i.e., in terms of the concentrations of the chemical substances being involved, making it clear from the outset that the model can provide at best only a rough approximation of the underlying molecular mechanisms.

The evolution of such a system over time is described by a system of differential equations (in the case in which the system is represented as a finite set of cells and their concentrations of the different chemical constituents) or by a system of partial differential equations (in the continuous case, where the system is represented via a set of continuous functions in space and time). The basic equations for Turing reaction-diffusion systems can be written as follows (cf. Murray 2003):

(1) $\frac{\delta c}{\delta t}=f(c)+D \nabla^{2} c$

where $c$ is a vector of morphogen concentrations, $f$ is the reaction kinetics and $D$ is the diagonal matrix of positive constant diffusion coefficients. The kinetics is, for all relevant situations, non-linear. The most common system studied mathematically is one involving two chemical substances (or species), with diffusion coefficients $D_{1}$ and $D_{2}$. In his mathematical analysis, Turing conjectured that if $D_{1}=D_{2}=0$ (diffusion is missing), $c$ tends to a linearly stable uniform steady-state. If, on the other hand, $D_{1} \neq D_{2}$, and certain other conditions are met, then spatially inhomogeneous patterns can evolve. Turing's analysis of the basic equations for a two-substance system is primarily a stability study. 
Equation (1) can also be presented in non-dimensional form, using the steady state of one of the morphogenes, and a typical length $(L)$ and time $(T)$ scales:

(2) $u_{t}=\gamma f(u, v)+\nabla^{2} u, v_{t}=\gamma g(u . v)+d \nabla^{2}{ }_{v}$

where $f$ and $g$ describe the non-linear kinetics and $u, v, \gamma$ and $d$ are determined via dimensional analysis. In order to calculate the solutions for these equations, one also needs to fix the initial and boundary conditions. Zero flux boundary conditions imply zero external input and thus are the most relevant for studying the formation of morphogen pattern in the absence of any chemical pre-patterns. The result of a linear analysis of equation (2), given initial and boundary conditions is a dispersion relation $\lambda\left(k^{2}\right)$, where $k$ is the wave number of a disturbance. Numerical simulations (carried out by hand in the case of Turing) allow one to predict and explore the precise shape of the Turing instabilities and their corresponding patterns. Turing himself identified 6 types of instabilities: uniform/stationary, uniform/ oscillating, stationary waves with extremely short wavelength, oscillatory cases with extremely short wavelength, oscillatory cases with finite wavelength, and stationary waves with finite wavelength (cf. Kondo and Miura 2010). However, as I will show in what follows, the question of their relevance for the biological domain is a separate issue from the stability analysis performed on the mathematical model and its associated technical challenges that arise when one considers the 3-dimensionality of the model or when RD systems are taken to involve more than 2 interacting substances.

The aim of the present section was to offer a broadly informal depiction of Turing's modeling strategy and results. According to Turing's theory, spatial patterns were the result of a process which he called "diffusion-driven instability". The analysis of his mathematical model showed that small spatial fluctuations in what was otherwise, by assumption, a well-mixed system of reacting and diffusing chemicals could become unstable. These fluctuations, Turing conjectured, could lead to a spatial pattern of chemicals that stimulated the development of form or structure in an organism. These are the morphogens. In other words, the spatial patterns of morphogens could serve as a pre-pattern for biological development. 
As mentioned above, two general features make Turing's mathematical model stand out: its simplicity (or minimality) and the way in which spatio-temporal patterns are mathematically generated in the model via a computational analysis of the characteristic state equations. In section 3 I return to show how these features can help us understand both the fruitfulness of Turing's modelling strategy and its mixed reception among experimental biologists.

\subsection{Extensions, challenges, and recent applications}

Although restrained to a single article, Turing's work on pattern formation has had a significant impact in both mathematics and biology. In the former domain, the focus fell on exploring the rich variety of behaviours of the system of nonlinear parabolic equations, while experimental work in biology targeted the discovery and detailed analysis of the structure of morphogens. The work of Hans Meinhardt and Alfred Gierer played a critical role in re-introducing and making relevant Turing's ideas in developmental biology. Their 1972 paper (cf. Meinhardt 1982) showed how to extend (or transform) the initially biologically unrealistic kinetics used by Turing in his modelling into more adequate kinetics that still preserved the central elements of his theory. This led to the formulation of the patterning principle known as 'short-range-activation, long-range-inhibition' (LALI). Its basic idea should be by now familiar from the analysis of Turing's model. If one morphogen activates another morphogen which, in turn, inhibits the first morphogen, then the ensuing state is one of equilibrium. But if one allows the inhibitor to diffuse more quickly than the activator, then this equilibrium is no longer stable, since the proposed mechanism leads to a local high concentration of activator, surrounded by a 'ring' of high inhibitor concentration. This corresponds to a specific type of pattern. Exploring exactly what types of complex patterned structures might arise requires, as Turing had shown, a combination of mathematical analysis and numerical simulation. Consistent with this feature of Turing's modelling strategy, Meinhardt's general patterning principle played a key role in the experimental discovery and investigation of Turing-like morphogen pairs. Meinhardt's own papers present several examples, including phyllotaxis, segmentation, veins, and pigmentation patterns in sea shells (Meinhardt et al. 2003). 
An even more remarkable feature of the LALI patterning principle is its compatibility with several mechanistic hypotheses about the processes underlying and driving morphogenesis. For instance, Oster and Murray's mechanochemical theory of morphogenesis (cf. Murray 2003) differs both mathematically and biologically from Turing's theoretical proposal. According to the mechanochemical theory, cells move through the extracellular material exerting large traction forces which deform it, and through the ensuing combination of active and passive transport processes, cells form different types of aggregates. Stripe formation in the visual cortex has been accounted in terms of neuronal firing mechanisms described by integro-partial differential equations, but this hypothesis is also compatible with LALI being the dominant physical principle at play in these patterning processes.

The compatibility of general patterning principles like LALI with multiple biological hypotheses raises a first serious challenge for the utility of such mathematical models in biology. In brief, the principle and its underlying justification cannot play a role in mechanistic model selection. If all the different mechanistic models produce the same types of patterns, one cannot use the general patterning principle to distinguish between candidate models. However, there is also a positive spinoff from this first problem for Turing-like models. This "insensivity" of this class of equations to the details of the patterngenerating biological mechanisms allows scientists to formulate hypotheses about the physical and biological boundaries within which certain types of patterning phenomena will occur. These have also been characterized as developmental constraints and are often described as "rules" that a biological system (or organism) must follow in the course of development, irrespectively of its particular biological constitution (e.g., Murray 2003, Maini 2012).

A set of related, and perhaps more important challenges concerns the "behaviour" of Turing-like models when biological details are added to the picture. One of the earliest applications of Turing's theoretical ideas was to pattern formation in developing Drosophila fruit fly. Experimental investigations led to the conclusion that the patterning in embryonic processes was actually due to a complex cascade of morphogen interactions. These results made many biologists wary of using Turing's theoretical hypothesis in the explanation 
of biological patterning. Even more sympathetic approaches had to admit that the model can be very sensitive to small changes in parameter values, initial morphogen fluctuations, and the geometry of the biological system in which pattern formation is studied. This put into question the ability of the model to produce, as robustly as it was initially suggested, the said spatial or spatio-temporal patterns (cf. Murray 2012, Maini et al. 2012).

Although definitive experimental proof that morphogens interact and pattern in the way Turing proposed is still lacking, a number of recent experimental studies may help to illustrate the epistemic potential of this modelling strategy and of its associated theoretical hypothesis for current biological research. Let us start with the Drosophila herself. In a recent study, Dilão (2015) has used Turing's morphogenesis hypothesis to show that in early stages of the development of the Drosophila, mRNA diffusion is the main (or dominant) morphogenesis mechanism which is able to account for the patterns of the experimentally determined gradients of relevant proteins. Dilão points out that the results of numerical simulations establish the steady nonuniform gradients of maternal and gap-gene families of proteins along the embryo of the Drosophila. Turing structures (patterns) have been shown to occur in chemical systems (Horvath et al. 2006, Tompkins et al. 2014) and a range of recent studies present evidence of their relevance in biological systems (eg. Othmer et al. 1992, Sick et al. 2006, Kondo and Miura 2010, Economou et al. 2012).

One of the most widely discussed studies is Sick et al. (2006) which investigated the regulation of hair follicle patterning in developing murine skin. They proposed two proteins as morphogens (WNT and its inhibitor DKK). Knowing that hair follicle patterning occurs in waves, the authors were justified to use a reactiondiffusion model to set up an initial pattern of follicles. They further assumed that these follicles were chemical sources giving rise to a second wave of hair follicle formation on a larger domain (due to growth of the skin). Their model predicted that the activator will be overexpressed, thus increasing follicular density, whereas moderate expression of the inhibitor during the initial wave will increase the interfollicular spacing. Having verified these predictions experimentally, Sick and colleagues concluded that their study provides strong evidence for a genetic basis of a Turing reaction-diffusion model. 
As mentioned above, an increasing number of studies ${ }^{1}$ offer converging evidence for the impact and utility of Turing's theoretical hypothesis and modelling technique. One note of caution though is in order to avoid too sweeping conclusions about the relationship between mathematical modelling and experimentation in fields such as developmental biology. Despite the agreement between the models' predictions and available biological experimental data, the studies do not rule out other potential mechanisms for producing the observed and simulated patterns. For instance, in the case of Sick et al.'s study, it has been argued that the follicle patterns can indeed be generated via a Turing-like process, but it is also possible for them to emerge without the type of chemical pre-pattern required by Turing's model (i.e., via simple chemotactic movement in response to gradients in chemical concentration). This brings us again to the first problem we noted with respect to Turing's model. Current experimental data cannot distinguish between candidate theoretical models. Maini and collaborators have pointed out that the next step in validating one or the other theoretical hypothesis requires overcoming the (primarily) 'experimental challenges in measuring key parameters (rates of production, decay, diffusion coefficients, etc.) so that quantitative tests can be performed to determine whether the system actually is of Turing type.' (Maini et al. 2006: 1398)

Before turning to the evaluation of the philosophical significance of this partial story of the relationship between mathematical modelling and experimental investigation in developmental biology, a brief word about the challenges facing the extension of the mathematical side of Turing's modelling technique. The Turing model has been studied primarily for the case of two interacting morphogens, where it has been shown to generate a wide array of spatial and spatiotemporal patterns. Even if these patterns have been studied numerically in detail, from an analytic perspective, the existing results are very limited. While pattern formation is linked to linear stability analysis, the nonlinear case supports only weakly the same type of connection. Extending the analysis to three or more interacting morphogens makes even the linear stability problem a challenge, which is currently solved only for a limited number of special cases. Rather than extracting a

${ }^{1}$ For an extended list of references, see Cooper and Maini 2012. 
general scepticism towards Turing's proposed set of equations, practicing mathematicians take them as sources of motivation for developing new techniques for analysing their behaviour (i.e., the emerging patterns) under a wider range of conditions, some of which might be relevant in a natural or in a synthetic biological setting.

\section{How Turing models explain}

How can we defend the claim that minimal models like Turing's reaction diffusion model can be used to provide adequate explanations of aspects of biological development? For this we need to specify first what counts as the explanandum, the explanans, and the explanatory connection established by Turing's minimal model. In addition, we should say something about the standards of explanation that apply in the relevant research context. In this case, the standards are related to the scientific knowledge available when Turing was developing his model and the reliability of the mathematical techniques being used.

Turing himself took the target of the explanation to be the phenomenon of morphogenesis, i.e., the generation of the shapes and patterns observed in living organisms. But closer scrutiny of the simplifying and idealizing assumptions on which the model was based leads to the identification of a more modest explanandum, viz. biological pattern formation. The explanans consists in the postulated interaction between two types of processes: chemical reactions and diffusion. The stability analysis performed on the system of coupled differential equations that describe this type of interaction yields a set of solutions corresponding to different types of spatio-temporal patterns with observable analogs in biological systems. Lastly, the derivation of the solutions of the system of state equations corresponds to the explanatory connection between the explanandum and the explanans. These three elements help us pin down the structure of the explanation based on Turing's mathematical model, but they still leave open the question about the biological relevance (or adequacy) of the proposed explanation.

It is not hard to see how this relevance issue might arise. After all, in delimiting the explanandum or target of his mathematical model, Turing left out almost all biological features of the living cell. Instead his model works with two minimal assumptions: a 
geometrical assumption about the spherical shape of the cell and a chemical assumption concerning the substances that interact within the cell during developmental processes. Even the reaction kinetics Turing did use in the model were not premised on any experimental data. Instead, the simplest chemical reactions were used to secure the tractability of the mathematical calculations. Claiming that the explanatory value of the model can be vindicated solely in light of the similarity between the computed solutions and some types of patterns observed during development seems too weak. So, can we justify the explanatory value of Turing's minimal model?

The answer that Turing's model provided to the question of how patterns arise during the development of a biological system was compatible with two key theoretical assumptions accepted at the time about biological development: (i) genetic conservation under cell division, and (2) the absence of causally effective inhomogeneities in the egg's cytoplasm. Notice that the idealizations introduced by Turing to model the developing embryo were compatible with these two key assumptions. Thus, even if the target of Turing's model was an imaginary system of reacting diffusing substances, there was a way to link it back (even if only minimally) to the real biological system. Turing's minimal explanation was thus in line with the available standards of biological knowledge. This general agreement, together with the reliability of the mathematical techniques employed support the claim that Turing's model provided a clear plausible description of the basic physical processes involved in biological pattern formation. Not only was Turing's explanation a how-possible or how-plausible account of the processes governing pattern formation, but it was the only physically viable account derivable from the background knowledge available at the time about biological development.

So far, we have made a case for the explanatory value of Turing's model, focusing on its minimality. We still need to clarify the epistemic contributions that mathematics makes to this explanatory result. First, mathematical concepts and techniques (operations) play a descriptive role in specifying the explanandum, the explanans and the derivation that connects them. Second, mathematics helps structurally by allowing the formulation of the explanation at an appropriate level of generality (cf. Yablo 2012). To clarify further the latter type of contribution we can turn to two of the applications of Turing's 
mathematical model mentioned in section 2.

As a first example consider Murray's reaction-diffusion models (Murray 2003) of the formation of patterns on the coats of various mammals (leopard spots and zebra stripes). In Murray's models, the activator induces melanocytes (cells that are responsible for the pigmentation of the epidermis) to produce melanin (pigment), while the repressor inhibits the production of the melanin. The explanandum in this case is the variation in pigmentation patterns that occurs despite the fact that similar processes (involving the same kinds of cells and many of the same proteins) are involved in coat pattern formation. The corresponding explanans is a reaction-diffusion system that also includes a parameter standing for the geometry of the organisms compared. Differences in the geometries of bodies are represented in the parameters of scale that yield different equilibria for the diffusion of the same type of molecules. The derivation shows that the same reaction diffusion process can yield different coat patterns depending on the choice of scale parameters. Note that Murray's explanatory minimal models require only the specification of the basic properties of activators and inhibitors, the initial concentration of the activator and the inhibitor, the scale parameters of the system and the differential equations describing the diffusion of molecules. More specific biochemical properties, such as the types of genes involved or the structure of the protein chains are not required to derive the relevant patterns. It is in this sense that the mathematical features of the model play a structural role: they enable the formulation of the explanation at an adequate level of generality.

A similar lesson can be drawn from Meinhardt's (1982) model of sea shells patterning. In this case, it is the cellular interactions which determine shell coiling that are modeled in terms of activatorinhibitor systems and rates of diffusion. Unlike in the case of coat patterning, which involves the same kinds of cells in most mammals, pigmentation patterns and relief patterns in sea shells are produced by different types of cells and proteins. However, reaction-diffusion equations can be applied to model and explain the similarities observed in coiling phenomena. Abstracting away from the specific identity of the morphogens that diffuse and interact with one another, and focusing on the geometry of the field over which the morphogens diffuse, the model can be used to derive the types of patterns 
observed in many types of sea shells. Thus, while Murray's minimal model aims to explain the variation of outcomes of similar biochemical processes, Meinhardt uses the same type of model to explain similarities in patterns produced by different biochemical processes. The explananda of the two models are different, but the explanans is the same: a reaction-diffusion system which abstracts away the biochemical properties and identity of the proteins involved. Therefore, Murray's minimal model explanation establishes how different systems can use very similar processes to produce different patterning outcomes, whereas Meinhardt's explanation shows that the same patterning effect can be obtained via a series of different causal pathways.

Despite their differences, the minimal model based explanations discussed in this section make up a distinctive class in the explanatory repertoire of developmental biology. Turing reaction-diffusion models can be viewed as an instance of minimal mathematical modeling that aims to uncover the dominant physical processes that are responsible for the generation of complex biological phenomena (cf. Batterman 2002, Weisberg 2007, Love and Nathan 2015). I suggest that the output of Turing's modelling strategy is best interpreted as establishing minimal internal constraints on the processes that lead to the development of specific spatial and spatio-temporal shapes in different biological organisms (cf. Amundson 2005).

It is tempting at this point to construct minimal-model-based explanations as a subspecies of causal explanations. After all, the common explanans of the explanations analyzed above refers to the physical processes that determine or govern the production of biological patterns. The terms involved in the specification of the explanans bear, at least semantically, the "mark" of causation. So are these minimal explanations a type of causal explanation or should they be conceived as a class of non-causal abstract explanations (Pincock 2015, Baron and Colyvan 2016)?

One reason to resist calling minimal explanations causal has to do with the way we have described the explanatory connection postulated by these minimal models. But here we must be careful not to mistake what belongs to the model with what belongs to the target phenomenon. It is true that the mathematical derivation links the explanandum to the explanans in the model, but no corresponding claim has been made about the link between pattern formation and the 
reaction-diffusion processes that the model postulates as the dominant physical factors in the world. On the other hand, it would also be misleading to construct minimal explanations as a subspecies of material causal explanations (Fox Keller 2003, Love 2008). Material causal explanations appeal to specific mechanisms or entities (specific genes, proteins etc.) to explain the occurrence of a particular (type of) effect. But these are not the only types of causal explanations encountered in the sciences. Structural causal explanations specify the conditions or constraints under which a certain type of effect (e.g. pattern formation) occurs. ${ }^{2}$ The explanations based on Turing-like reaction-diffusion models specify such minimal constraints on the processes involved in biological pattern formation. As our analysis showed, these explanations can stand on their own feet in specific research contexts where they provide adequate answers to certain explanatory questions. Other times, they can also figure as partial accounts of a more complete causal account of morphogenesis. In this sense, it seems legitimate to talk about the explanatory contributions that such minimal models make to the broader investigation of biological morphogenesis.

A different classificatory scheme can help us sharpen the thesis about the explanatory value of these minimal models. Marco Nathan (2012) calls reaction-diffusion based explanations, morphological explanations because they focus solely on the form or shape properties of biological systems. He distinguishes them from genetic explanations which cite, as explanatory factors, structural and functional properties of nucleic acids and proteins. In contrast, the explananda of morphological explanations are properties of biological systems specified at a certain level of generality, while their explanantia involve geometrical or scale properties that determine the shape of the solutions

\footnotetext{
${ }^{2}$ Another way to pin down the contrast relevant for the two types of causal explanations is Dretske's distinction between triggering causes and structuring causes: 'In looking for the cause of a process, we are sometimes looking for the triggering event: what causes the $C$ which caused the $M$. At other times we are looking for the event or events that shaped or structured the process: what caused $C$ to cause $M$ rather than something else. The first type of cause, the triggering cause, causes the process to occur now. The second type of cause, the structuring cause, is responsible for its being this process, one having $M$ as its product, that occurs now ....' (Dretske 1988: 42)
} 
to the postulated reaction-diffusion equations. A third type of explanation encountered in developmental biology are morphogenetic explanations which combine material and structuring causal information to answer certain questions about developmental processes. One example of the latter type of explanation is the account of axis formation in Drosophila sketched in section 2. Minimal internal constraints (geometrical properties of the system and dispositional properties of reactants) are invoked in this account via the reaction-diffusion model employed to answer explanatory questions about segment specification in Drosophila. However, molecular information about the specific genes involved (hemoglobin) is also required to explain the segmentation process. That is, the parameters that control what happens with each nucleus include both the interactions of genes and gene products and the spatial organization of the embryo that imposes constraints on the concentration of morphogens in the oocyte. As Nathan points out: 'Both molecular details and geometrical properties of the system are necessary to explain the segmentation of Drosophila, but neither is, by itself, sufficient' (Nathan 2012: 248).

I have argued that explanations based on minimal models such as Turing's reaction-diffusion models can account for certain morphological features of biological systems. They provide information about the minimal internal constraints or structuring causes that are responsible for the occurrence of certain types of patterns. ${ }^{3}$ Minimal explanations can be combined or coordinated with other types of explanatory information yielding morphogenetic explanations of more complex targets such as the developmental process of axis formation. The take home message here is that the explanatory contributions of such minimal models is not cancelled out by the possibility to embed, extend or otherwise alter the original minimal models to construct other types of biological explanations. The standards of explanatory satisfaction applicable to the evaluation of

\footnotetext{
${ }^{3}$ It is worth pointing out that conceiving minimal explanations as structural causal explanations is compatible with several philosophical accounts of the nature of causation. In particular, the proposed account is friendly to Woodward's interventionist notion of causation (Woodward 2003). However, clarifying the link between the two conceptions would require a separate investigation, especially since none of the applications of Turing minimal models discussed in section 2 directly illustrates the interventionist methodology on which Woodward's conception is based.
} 
morphological explanations might be different from those applicable to the evaluation of genetic or morphogenetic explanations, but they are epistemically on a par.

\section{A functionalist perspective on explanation}

Our analysis so far has focused on clarifying the structure of minimal explanations of biological pattern formation and identifying the explanatory satisfaction (or adequacy) conditions applicable to this family of explanations. But there is a broader lesson about scientific explanation to be extracted from the survey offered in section 2 . The question that can help us unlock it is: What are the roles (or epistemic functions) that explanations play in scientific practice? Thinking about the roles that explanations play in science allows us to articulate the links between explanation and other epistemic outcomes of scientific research, such as description, generalization, prediction, and control, etc. Andrea Woody (2015) has used a similar question to promote a reorientation of philosophical debates about scientific explanation that would correspond to a more adequate descriptive account of the explanatory activities of practicing scientists. I agree with Woody that the functional perspective is very important in philosophy of science, but my aims are more modest than reformulating the debates about the problem of explanation. I believe that the functional perspective can help strengthen some of claims made about the explanatory value of minimal models by placing them in a broader epistemic landscape.

The following is not intended as an exhaustive list of the epistemic roles that explanation may play in scientific practice. It is also a "biased" list in the sense that it is derived from the survey provided in section 2. With these caveats in place, we can point to the following roles of explanation: providing standard descriptions of the objects of scientific investigation, communicating the results of different modelling techniques, establishing links between different domains of scientific knowledge, creating templates of intelligibility for specific scientific domains, facilitating the coordination between theoretical hypotheses and experimental investigation, enabling further applications of the models or modelling techniques on which the explanation is based, and providing exploratory tools for new topics 
of scientific investigation. Next I aim to illustrate how focusing on some of these roles can help us better understand the links between the epistemic uses of minimal models.

First, note that Turing's minimal mathematical model is highly modular in that it can be extended in various ways, viz. by specifying different reaction kinetic functions and considering different parametrizations of the characteristic differential equations. Such modified versions of Turing's reaction-diffusion model have been used in recent studies to derive and study, via numerical simulations, a wider range of patterns that might be relevant in natural and synthetic biological settings. These simulations have yielded a wide range of predictions which are compared with experimental data about naturally occurring biological patterns. In light of these observations, we can claim that Turing's reaction diffusion models provide standardized descriptions of some of the targets investigated in developmental biology: different types of spatial and temporal patterns. They also have been used as exploratory tools to investigate a wide range of biological systems at different levels of organization. The modularity of Turing's models has been a key factor in encouraging these additional applications which in turn have led to the creation of stronger connection between biological theorizing and experimental investigation of developmental systems. These functions of the explanations based on Turing's models allow us to see the connections between the explanatory, predictive, and control uses of this class of models. The extensions of Turing's reaction-diffusion model made possible novel predictions about specific causal factors involved at different stages in the developmental process. Because they provide an intelligibility template in developmental biology, reaction diffusion models can be used (albeit in a limited way) for the purpose of mechanism comparison and analysis.

Marking the connection between the different epistemic virtues of a class of models is not a novel idea in philosophy of science. The novelty resides in the way in which this connection has been introduced. I suggested that when one focuses on the roles or epistemic fuctions that explanation plays in science, it is possible to show that having a minimal explanation can sometimes increase the descriptive, predictive or control power that scientists have in specific research contexts. Admitting to a more dynamic interplay between the epistemic 
virtues of minimal models motivates a more general diachronic view of the process of scientific explanation to which I now turn.

\section{Towards a diachronic study of scientific explanation}

Some models are good predictors and bad explainers, others explain without producing novel predictions, while yet other models are fruitful heuristic tools aiding in the formulation or refinement of experimental and theoretical hypotheses. Surely we should allow our scientific models to be more promiscuous than the theories which we enlist in our repository of trusted scientific knowledge. So why insist on the complementarity of the epistemic functions of scientific, or more specifically in this case, of minimal mathematical modelling?

The rationale is simple and it does not directly conflict with the intuition encapsulated in the previous remarks. It amounts to the request for an epistemological account that would make sense of the productive interactions between experimental, theoretical, and mathematical modelling currently pursued in many fields of biology (Brigandt 2013). While evidential and, more broadly, methodological opportunism might recommend the adoption of multiple modelling techniques for their more immediate epistemic gains, a stronger normative desideratum is to recruit those models which can fulfill more than one epistemic function since this would facilitate a smoother integration of the different approaches that target the understanding of the same biological phenomena (Love and Lugar 2013, Love and Nathan 2015).

I propose that a diachronic view of model-based explanations provides a good framework for thinking about how different explanations might be coordinated or integrated in different areas of biological research. The core idea is that explanations, in science, just as in other areas of human inquiry, are dynamic. They can be adapted and transformed to fit the other epistemic activities that we deem worthwhile pursuing as part of scientific research. Is this dynamic picture of explanation unstable or unsystematic? No. It admits a variability in explanatory judgments that is driven by various epistemic and nonepistemic factors, but not at the price of trivialization. Any philosophical account of the structure of explanation should include the fact that an explanation provided in a specific context of inquiry selects 
and presents in an adequate representational format a dependence relation that holds between the feature or behaviour of the target system identified as the explanandum, and the explanans. These dependence relations might be construed as: constitution relations that specifies the components parts and properties of the system taken as the explanandum, general lawful statements that subsumes the explanandum, or material or structural causal relations (cf. Pincock 2015). Different philosophical models of scientific explanation typically spell out in more detail the nature of the explanatory dependence relation. This essay has attempted to do the same for minimal explanations based on the class of reaction-diffusion models originally proposed by Turing. It has been argued that these models provide structural causal information that links the explanandum (biological patterns) to the explanans (the interaction of reaction and diffusion processes).

The analysis of Turing's modelling strategy and its recent extensions and applications in developmental biology illustrates this diachronic view of explanation. Depending on the empirical questions being asked in different inquiry situations, the mathematical elements of Turing's model can be said to play a more or less central role in the construction of an adequate biological explanation. Turing models can be both explananda and explanantia, depending on the type of inquiry one engages in. Numerical simulations of extended and refined Turing models of various biological patterning processes enable the accumulation of novel predictions that need to be compared and assessed against experimental data. Mathematical analyses of Turing-like systems of parabolic equations might suggest new causal hypotheses about the processes underlying pattern formation in different types of systems. The investigative paths pursued in developmental biology support the contention that Turing models can serve multiple epistemic functions in biological research, which foster further collaborations between mathematical and mechanistic methodologies in the investigation of biological phenomena.

\section{Conclusion}

What explains the resilience of minimal mathematical models, such as Turing's model of pattern formation, in empirical research carried out in the life sciences? I have argued that minimal mathematical 
models can identify and test hypotheses about the dominant physical features of the phenomena biologists are trying to explain and understand. In some research contexts, these features can be viewed as internal constraints on the local mechanisms that produce the observed biological phenomena. Such minimal mathematical models also aid in structuring the epistemic practices of biology by providing precise descriptions of the quantitative relations between various features of the complex systems, by generating novel predictions that can be compared with experimental data, by promoting theory exploration, and by acting as constitutive parts of empirically adequate explanations of naturally occurring phenomena, such as biological pattern formation. Focusing on the roles that minimal model explanations play in science motivates the adoption of a broader diachronic view of scientific explanation.

Maria Serban

Department of Media, Cognition and Communication Philosophy Section, University of Copenhagen Karen Blixens Vej 4

Copenhagen 2300

Denmark

mariaserban.org@gmail.com

\section{References}

Amundson, Ron. 2005. The Changing Role of the Embryo in Evolutionary Thought: Roots of Evo-Devo. Cambridge MA: Cambridge University Press.

Baker, Alan. 2005. Are there genuine mathematical explanations of physical phenomena? Mind 114 (454): 223-38.

Baker, Alan. 2009. Mathematical explanation in science. British Journal for the Philosophy of Science 60 (3): 611-33.

Batterman, Robert. 2002. Asymptotics and the role of minimal models. British Journal for the Philosophy of Science 53 (1): 21-38.

Baron, Sam; and Colyvan, Mark. 2016. Time enough for explanation. Journal of Philosophy 113(2): 61-88.

Bechtel, William P.; and Abrahamsen, Adele. 2010. Dynamic mechanistic explanation: computational modeling of circadian rhythms as an exemplar for cognitive science. Studies in History and Philosophy of Science Part A 41(3): 321-33.

Bechtel, Wiliam P.; and Richardson, Robert C. 2010. Discovering Complexity Decomposition and Localization as Strategies in Scientific Research. $2^{\text {nd }}$ edition. 
Cambridge MA: MIT Press.

Bechtel, William P. 2015. Can mechanistic explanation be reconciled with scalefree constitution and dynamics? Studies in History and Philosophy of Science Part C: Studies in History and Philosophy of Biological and Biomedical Sciences 53: 84-93.

Bourgine, Paul; and Lesne, Annick. 2006. Morphogenesis: Origins of Patterns and Shapes. Berlin: Springer Verlag.

Brigandt, Ingo. 2010. Beyond reduction and pluralism: Toward an epistemology of explanatory integration in biology. Erkenntnis 73 (3):295-311.

Brigandt, Ingo. 2013. Systems biology and the integration of mechanistic explanation and mathematical explanation. Studies in History and Philosophy of Biological and Biomedical Sciences 44(4): 477-92.

Cooper, S. Barry; and Maini, Philip K. 2012. The mathematics of nature at the Alan Turing centenary. Interface Focus 2: 393-6.

Craver, Carl F.; and Darden, Lindley. 2013. In Search of Mechanisms: Discoveries across the life sciences. Chicago: University of Chicago Press.

Dilão, Rui. 2015. Mathematical models of morphogenesis. ITM Web of Conferences 4.

Dretske, Fred. 1988. Explaining Behavior: Reasons in a World of Causes. Cambridge MA: MIT Press.

Economou, Andrew et al. 2012. Periodic stripe formation by a Turing mechanism operating at growth zones in the mammalian palate Nature Genetics 44: 348-51.

Fox Keller, Evelyn. 2003. Making Sense of Life: Explaining Biological Development with Models, Metaphors, and Machines. Harvard University Press.

Horvath, Judit; Szalai, Istvan; and De Kepper, Patrick. 2009. An experimental design method leading to chemical Turing patterns. Science 324: 772-5.

Kondo, Shigeru; and Miura, Takashi. 2010. Reaction-diffusion model as a framework for understanding biological pattern formation. Science 329(5999): 1616-20.

Lange, Marc. 2013. What makes a scientific explanation distinctively mathematical? British Journal for the Philosophy of Science 64 (3): 485-511.

Levy, Arnon. 2015. Modeling without models. Philosophical Studies 172 (3): 781-98.

Love, Alan. 2008. Explaining the ontogeny of form: philosophical issues. In A Companion to the Philosophy of Biology, ed. by Sarkar Sahorta and Anya Plutinski. Oxford: Wiley Press.

Love, Alan C.; and Lugar, Gary L. 2013. Dimensions of integration in interdisciplinary explanations of the origin of evolutionary novelty. Studies in History and Philosophy of Science Part C: Studies in History and Philosophy of Biological and Biomedical Sciences 44(4): 537-50.

Love, Alan C.; and Nathan, Marco J. 2015. The idealization of causation in mechanistic explanation. Philosophy of Science 82(5): 761-74.

Madzvamuse, Anotida; Gaffney, Eamonn A.; and Maini, Philip K. 2010. Stability analysis of non-autonomous reaction-diffusion systems: the effects of growing domains. Journal of mathematical biology 61(1): 133-64 PMID: 19727733.

Maini, Philip K.; Baker, Ruth E.; and Chuong, Cheng-Ming. 2006. The Turing model comes of molecular age. Science Developmental Biology 314: 1397-8.

Maini, Philip K. et al. 2012. Turing's model for biological pattern formation and 
the robustness problem. Interface Focus 2: 483-496.

Maini, Philip K. 2012. Turing's mathematical theory of morphogenesis. Asia Pacific Mathematics Newsletter 2(1): 7-8.

Meinhardt, Hans. 1982. Models of Biological Pattern Formation. London: Academic London.

Meinhardt, Hans et al. 2003. The Algorithmic Beauty of Sea Shells, 3rd edition. New York: Springer.

Murray, James D. 2003. Mathematical Biology II: Spatial Models and Biomedical Applications. Springer USA.

Murray, James D. 2012. Vignettes from the field of mathematical biology: the application of mathematics to biology and medicine. Interface Focus 2: 397-406.

Nathan, Marco J. 2012. The varieties of molecular explanation. Philosophy of Science 79(2): 233-54.

Othmer, Hans G.; Maini, Philip K.; and Murray, James D. 1993. Experimental and Theoretical Advances in Biological Pattern Formation. Springer USA.

Pincock, Christopher. 2007. Mathematical idealization. Philosophy of Science 74 (5): 957-967.

Pincock, Christopher. 2011. Mathematics and Scientific Representation. Cambridge MA: Oxford University Press.

Pincock, Christopher. 2012. Mathematical models of biological patterns: lessons from Hamilton's selfish herd. Biology and Philosophy 27(4): 481-96.

Pincock, Christopher. 2015a. The unsolvability of the quintic: a case study in abstract mathematical explanation. Philosophers' Imprint 15(3).

Pincock, Christopher. 2015b. Abstract explanations in science. British Journal for the Philosophy of Science 66 (4): 857-82.

Raz, Tim; and Sauer, Tilman. 2015. Outline of a dynamical inferential conception of the application of mathematics. Studies in History and Philosophy of Science Part B: Studies in History and Philosophy of Modern Physics 49: 57-72.

Tompkins, Nathan et al. 2014. Testing Turing's theory of morphogenesis in chemical cells. PNAS 111(12): 4397-402.

Turing, Alan M. 1952. The chemical basis of morphogenesis. Philosophical Transactions of the Royal Society of London. Series B, Biological Sciences DOI: 10.1098/rstb.1952.0012.

Weisberg, Michael. 2007. Three kinds of idealization. Journal of Philosophy 104 (12): 639-59.

Winther, Rasmus G. 2012. Mathematical modeling in biology: philosophy and pragmatics. Frontiers in Plant Evolution and Development 2012: 1-3.

Woody, Andrea. 2015. Re-orienting discussions of scientific explanation: a functional perspective. Studies in History and Philosophy of Science Part A 52: 79-87.

Yablo, Stephen. 2012. Explanation, extrapolation, and existence. Mind 121 (484): 1007-29. 